\title{
Numerical Simulation of Excitation-Contraction in Isolated Cardiomyocytes
}

\author{
José Alberto Rodrigues ${ }^{1,2, *}$, Joana Oliveira ${ }^{3}$ \\ ${ }^{1}$ Department of Mathematics, Instituto Superior de Engenharia de Lisboa-ISEL, Portugal \\ ${ }^{2}$ GIMOSM, ISEL, IPL Polytechnic institute of lisbon \\ ${ }^{3}$ Biomedical Engineering, Instituto Superior de Engenharia de Lisboa-ISEL, Portugal
}

\begin{abstract}
We present and study a mathematical model to simulate the calcium flux and contractile activity of the cardiomyocyte, resorting on the Finite Element Method. This Model of cardiac cells provide the possibility to understand the biochemistry and biomechanics of cardiac cells and cardiovascular diseases. Heart Failure is considered the ultimate cardiac disease, a condition with no effective cure which is highly related with the function of cardiomyocytes and consequently with the concentration of calcium, affecting the contractile activity of the cardiomyocyte.

In order to test our model's performance, several tests were applied varying the local active cellular tension driven by the intracellular calcium concentration and the localization of the main calcium influx. The results are expressed in the graphics of calcium concentration over time, maximum cardiomyocyte contraction and the gradient of calcium diffusion.

Our results show that the behaviour of our models is faithful to what is known to be true with cell's physiology and pathological conditions.
\end{abstract}

Keywords Biomedical Engineering, $\mathrm{Ca}^{2+}$ Oscillations, Mechano-chemical Mathematical Model, Finite Element Methods.

AMS 2010 subject classifications 65M60, 92B05, 92C10

DOI: $10.19139 /$ soic.v7i3.835

\section{Introduction}

The recent evolution in biotechnology and informatics has promoted the production of large amounts of information and has accelerated the processes of investigation in biological systems and cellular-based studies. The use of mathematical and geometric models enables the simulation of biological complex systems, and therefore, the formulation of hypotheses and realisation of experiments that would normally require the use of live cells and its lifetime conditions.

Models of cardiac cells are capable of facilitating insights into the mechanisms underlying cardiac dynamics and its pathophysiology. They provide the possibility to understand the biochemistry and biomechanics of cardiac cells, since the cardiovascular diseases have reached an enormous burden on contemporary society. In fact, Heart Failure, the ultimate cardiac disease which has no effective cure, has been increasing in mortality and morbidity[1], and its evolution always has a reserved prognosis. From this very negative trend, the need arises to create and test a new tool that could be an added value in the study of the biomechanical mechanisms of the cardiomyocyte: numerical modelling.[2]

In this work, the numerical modelling uses equations, discretized by the Finite Element Method, which model the known cellular dynamics, as well as the possible dysfunctions. Thus, it becomes possible to create and to experiment with other hypotheses, with unlimited resources, and without raising ethical questions.

\footnotetext{
*Correspondence to: José Alberto Rodrigues (Email: jrodrigues@adm.isel.pt). Department of Mathematics, Instituto Superior de Engenharia de Lisboa-ISEL,Rua Conselheiro Emídio Navarro, 1959-007 Lisboa, Portugal.
}

ISSN 2310-5070 (online) ISSN 2311-004X (print)

Copyright (C) 2019 International Academic Press 
The main objective of this work is to simulate the calcium efflux and contractile activity of the cardiomyocyte, resorting on the finite element method. When this becomes a successful and working model, there will be the possibility to promote the reduction of in vivo/in vitro experimentation.

\section{The Cardiomyocyte}

The human heart is an essential organ with a highly organized tissue constituted of ventricular and atrial cardiomyocytes, pacemaker cells, Purkinje cells, vasculature, and connective tissue. Cardiomyocytes are mononuclear cells and form the striated muscle of the heart, with the shape of a tridimentional net. This tridimentional structure allows the heart to contract and relax in the most efficient way. The ventricular cardiomyocytes are columnar shaped cells of $20 \mathrm{~nm}$ in diameter and $60-140 \mathrm{~nm}$ in length, while the atrial cardiomyocytes are ellipsoidal shaped cells of $5 \mathrm{~nm}$ in diameter and $10-20 \mathrm{~nm}$ in length. [3]

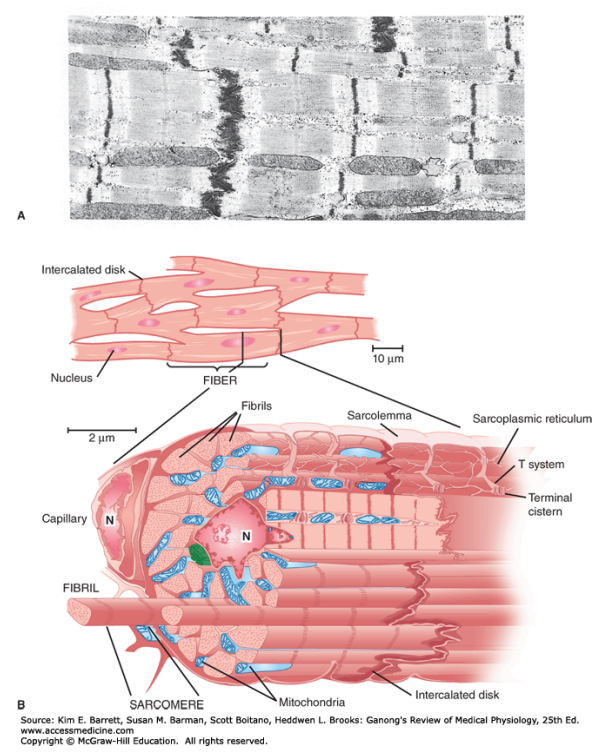

Figure 1. Morpholohy of Cardiac Muscle

[4]

The biggest part of the whole cell volume $(75 \%)$ is made up of myofibrils and mitochondria. Myofibril is the bundle that forms the contractile elements within the cell and it is composed of actin thin filament, myosin thick filament and titin, which stabilizes myosin at the Z-line. The fundamental unit within the cardiomyocyte that allows its contraction is the sarcomere (aggregation of myofibrils), which has a length of $1.8 \mu \mathrm{m}$ in the systole and $2.2 \mu \mathrm{m}$ in the diastole.

Other than myofibrils, this contractile structure contains tropomyosin (troponin complex). Myosin has a filamentous tail and a globular head region that contains the site for actin binding. Actin has F-actin, the backbone of the thin filament, and G-actin, which works as a stabilizing protein.

The Sarcoplasmic Reticulum (SR), the nucleus, and the cytosol occupy the other $25 \%$ of the cell. Surrounding these components, there is a specialized structure called the Sarcolemma, which is a coalescence of the plasma membrane and the basement membrane, composed of a lipid bilayer. This wall contains hydrophilic heads and hydrophobic tails, allowing the regulation of the interactions with the intracellular and extracellular environment. In order to maximize the in and out interactions, there are transverse tubular systems (T-tubules) in the sarcolemma.

The T-tubules are $150 \mathrm{~nm}$ to $300 \mathrm{~nm}$ wide deep invaginations of the sarcolemma into the cardiomyocyte, and they form an extended barrier between the intracellular and extracellular space, turning them into important structural components in the Excitation-Contraction Coupling (ECC) system. 
All of these cell organelle interactions are included in the ECC process and are fuelled by the flow of three important ions: $\mathrm{K}^{+}, \mathrm{Ca}^{2+}$ and $\mathrm{Na}^{2+}$.

\subsection{Structural details}

The Ryanodine Receptor (RyRs) are ion channels that open in the presence of elevated $\mathrm{Ca}^{2+}$ and release calcium from the junctional sarcoplasmic reticulum. The junctional sarcoplasmic reticulum makes such close contact with the T-tubule membrane that RyRs channels on the sarcoplasmic reticulum are impending apposed $(\approx 15 \mathrm{~nm})$ to L-type $\mathrm{Ca}^{2+}$ channels on the T-tubule $(\approx 14$ in peripheral couplings to $\approx<100$ in intracellular sites), this way forming the cardiac dyad that is fundamental to the processes of $\mathrm{Ca}^{2+}$ flow. from the junctional sarcoplasmic reticulum. When viewed on detail, T-tubules are seen to radiate throughout the cardiac cell. This close association between the T-tubule and sarcoplasmic reticulum ensures the synchronous rise of $\left[\mathrm{Ca}^{2+}\right]$, during systole, therefore, the importance of the whole architecture of the cell. In addition to these basic requirements for ECC process, the cardiac dyad may also be considered as containing additional structures that may contribute to or modulate $\mathrm{Ca}^{2+}$ release from the sarcoplasmic reticulum during systole. The most extensively studied is $\mathrm{Na}^{2+} / \mathrm{Ca}^{2+}$ eXchanger (NCX), that has been argued via its reverse-mode action to contribute to $C a^{2+}$ influx early during the action potential. The second protein of interest is SERCA2a: Sarco(Endo)plasmic Reticulum Calcium ATPase. Available data on SERCA2 $a$ distribution in the heart is surprisingly sparse; however, confocal studies seem to suggest that a significant proportion of SERCA is localized to the z-line as well as enveloping the myofilaments.[5] This cycle of calcium triggering, release and uptake, takes place in the approximately 20,000 diad spaces (also called calcium release sites) that are distributed throughout the myocyte. [6]

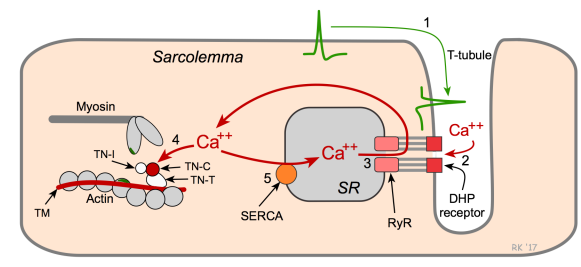

Figure 2. Close look to cardiac diad

[7]

\section{From Action Potential to Contraction}

ECC characterizes the whole processes relating to electrical excitation through force generation and the power of contraction in the heart. It occurs at multiple levels from the whole heart, to each single cardiomyocyte and down to the sarcomere.[6] The propagation of the action potential through the entire cardiac muscle requires the depolarization of a cardiomyocyte. This means that the cell will generate an action potential while arising from a steady state to an excited state, and then pass it to the neighbour cell. In order to induce the process, an electric charge will be propagated by the three cations already referred. These three ions are small enough to be widespread through the cells membranes. The contraction of individual myocytes is coordinated by a propagation wave of electrical depolarization of the sarcolemma.

The propagation from cell to cell of the action potential corresponds to the beginning of the action potential cycle (phase 0) and requires a difference between cell voltages. The bigger the difference, the bigger the flow between the cells. When the stimulus reaches a steady state cell, it becomes excited and some of the $\mathrm{Na}^{2+}$ channels open. The $\mathrm{Na}^{2+}$ moves in the cell, becoming less negative. When the cell voltage reaches $-70 \mathrm{mV}$, every $\mathrm{Na}^{2+}$ channels open and more $\mathrm{Na}^{2+}$ ions get in the cell. The cell becomes positive. The $\mathrm{Na}^{2+}$ channels close, making the cell more negative again. 


\subsection{Chemical aspects}

In the beginning of the contraction stimulus, the $K^{+}$channels open and $K^{+}$ions move out of the cell. This $K^{+}$ extraction, decreases the cell potential from $20 \mathrm{mV}$ to $0 \mathrm{mV}$ (phase 1). Ion $\mathrm{Ca}^{2+}$ is fundamental for evoking the ECC complex. Upon the wave of depolarization, when electrical action potential reaches T-tubules, the wave of depolarization induces $\mathrm{Ca}^{2+}$ influx into the cardiomyocyte through the voltage-sensitive L-type $\mathrm{Ca}^{2+}$ channel of the T-tubules. This rapid but small $\mathrm{Ca}^{2+}$ influx causes activation of large amounts of $\mathrm{Ca}^{2+}$ release from the RyR2 on the sarcoplasmic reticulum. Finally, cytosolic $\mathrm{Ca}^{2+}$ level changes from $100 \mathrm{nmol} / \mathrm{L}$ to $10 \mu \mathrm{mol} / \mathrm{L}$ in concentration. The $10 \mu \mathrm{mol} / \mathrm{L}$ of $\mathrm{Ca}^{2+}$ binds to the troponin $\mathrm{C}$, leading to the actin-myosin interaction resulting in initiating crossbridge formation and, therefore, contraction movement (phase 2).

Using Adenosine Tri-Phosphate (ATP), the G-actin interacts with the myosin globular head leading to the crossbridge formation and sarcomere shorting (contraction). Tropomyosin lies on the side of actin for rigidity to thin filament. The troponin complex, also present in the thin filament, is composed of troponin $\mathrm{C}$, I and T. These proteins are responsabile for the regulation of the crossbridge formation.

Active relaxation (phase 3 ) of the cardiomyocyte is dependent on the function of the SERCA2a. For each $1 \mathrm{~mol}$ of ATP hydrolysed, $2 \mathrm{~mol}$ of $\mathrm{Ca}^{2+}$ is transported back into the sarcoplasmic reticulum. Phospholamban (PLB) regulates the function of $S E R C A 2 a$. Additionally, the NCX on the plasma membrane removes $C a^{2}$ from cytosol, until the cell reaches a voltage of $-90 \mathrm{mV}$, changing $\mathrm{Ca}^{2+}$ for $\mathrm{Na}^{2+}$. In summary, the process of ECC links the electric excitation of the surface membrane, i. e. action potential, to contraction, with the concentration of ion $\mathrm{Ca}^{2+}$ being the major factor for the magnitude and duration of the contraction.

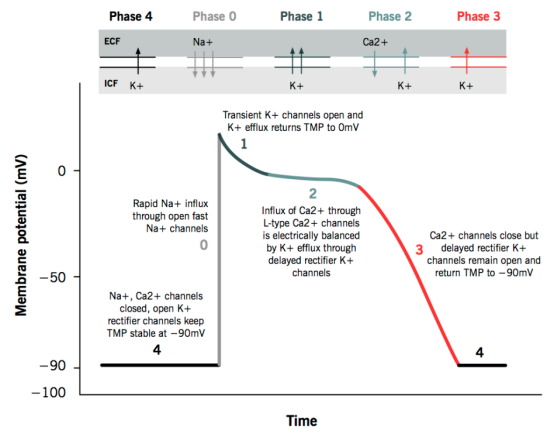

Figure 3. Action Potential in Cardiomyocytes

[9]

In the end, the purpose of cardiomyocytes is to generate a controlled contraction in response to repetitive electrical depolarization signals - a function that is fully controlled by $\mathrm{Ca}^{2+}$.

The importance of ion $\mathrm{Ca}^{2+}$ will be shown throughout this work, as the ion's flow will be used as an effective tool to understand the complex dynamics of the processes involved in excitation-contraction coupling and in its computational simulation.

\subsection{Mathematical model aspects}

We consider for a seek of simplicity the quite well established two variable model of Goldbeter et al. [11] as the core for self-sustained calcium oscillations (phases 2 and 3). In this model, the calcium-induced calcium release mechanism is described by nonlinear kinetics between cytosolic $c=c(t, x, y)$ and sarcoplasmic $s=s(t, x, y)$ calcium concentrations at instant $t$ and position $(x, y)$, with rates of variation given by the differential system:

$$
\begin{aligned}
& \frac{d s}{d t}=F(c, s) \\
& \frac{d c}{d t}=v_{0}-v_{1} \beta-k_{c} c-F(c, s)-\nabla \cdot(\mathbf{D} \nabla c)
\end{aligned}
$$


With

$$
\begin{aligned}
F(c, s) & =v_{2}-v_{3}-k_{s} s \\
v_{2} & =M_{1} \frac{c^{n}}{K_{2}^{n}+c^{n}} \\
v_{3} & =M_{2} \frac{s^{n}}{K_{R}^{n}+s^{n}} \frac{c^{p}}{K_{A}^{p}+c^{p}}
\end{aligned}
$$

The intracellular propagation of free calcium is taken into account in the model by considering the anisotropic diffusion of calcium observed experimentally at [13] with $\mathbf{D}=\left(D_{1}, D_{2}\right)$.

In this model, $v_{1} \beta$ is the constant flow of calcium into the cytosol, controlled by the sarcoplasmic reticulum. The concentration of free cytosolic calcium $c$ oscillates between low levels, during which $c$ is pumped by the sarcoplasmic reticulum ( $v_{2}$ flux), and high levels, characterised by the autocatalytic release of calcium $v_{3}$ from the sarcoplasmic reticulum. The flux $k_{s} s$ is a basal leak of calcium into the sarcoplasmic reticulum, while the input flux $v_{0}$ and efflux $k_{c} c$ refer to the $C a^{2+}$ fluxes into and out of the cell, respectively, finally $\mathbf{D}$ is the diffusion tensor with components $D_{i j}$.

Equations (1) and (2) describe an entire family of models dependent on the particular choice of the exchange function $F(c, s)$ that describes the dynamics of $C a^{2+}$ exchange between the cytosol and the internal $\mathrm{Ca}^{2+}$ pool. It should, therefore, be kept in mind that, although we present results here for a particular choice of $F$, similar results can be obtained from exchange functions with similar qualitative properties.

For the contraction movement (phase 2) we assume that the total stress $\boldsymbol{\sigma}=\sigma_{i j}$ within the cardiac cell is generated by the contraction of actin-myosine fibers. Since the sarcomeres are oriented along the cell principal axis ( $x$ axis), we consider an isotropic linear elastic motion governed by:

$$
\left\{\begin{array}{l}
\frac{\partial \sigma_{11}}{\partial x}+\frac{\partial \sigma_{12}}{\partial y}=-\gamma(c) T_{u} \\
\frac{\partial \sigma_{21}}{\partial x}+\frac{\partial \sigma_{22}}{\partial y}=0
\end{array}\right.
$$

where $\gamma(c) T_{u}$ is the local active cellular tension driven by the intracellular calcium concentration $c=c(t, x, y)$. We assume that the analytical expression of $\gamma$ is given by a Hill function of the form [14]:

$$
\gamma(c)=\frac{c^{4}}{\eta^{4}+c^{4}}
$$

\section{Finite element discretization}

Using Finite Element Method like in Biomedical Engineering Handbook Vol 1, Sec 12.3 [15] and Claes Johnson [16] equations (1),(2) and (6) assume respectively the integral form:

$$
\begin{aligned}
\int_{\Omega} \frac{d s}{d t} w d \Omega= & \int_{\Omega} F(c, s) w d \Omega \\
\int_{\Omega} \frac{d c}{d t} w d \Omega= & \int_{\Omega}\left(v_{0}-v_{1} \beta-k_{c} c-F(c, s)\right) w d \Omega+\int_{\Omega} \mathbf{D} \cdot \mathbf{D} \nabla c \cdot \nabla w d \Omega- \\
& \int_{\Gamma_{T}} D_{1} n_{x} \frac{\partial c}{\partial x} w+D_{2} n_{y} \frac{\partial c}{\partial y} w d \Gamma \\
\int_{\Omega} \lambda \operatorname{div}\left(u_{x}, u_{y}\right) \operatorname{div}\left(v_{x}, v_{y}\right)+ & \mu \varepsilon\left(u_{x}, u_{y}\right): \varepsilon\left(v_{x}, v_{y}\right) d \Omega=\int_{\Omega} \gamma(c) T_{0}\left(v_{x}, v_{y}\right) \cdot \vec{e}_{x} d \Omega
\end{aligned}
$$


where $\left(n_{x}, n_{y}\right)$ is the outward unit normal to $\Gamma_{T}$.

We remark that at equation (10) we have used the Hooke's law for plane stress, with the displacement vector $\left(u_{x}, u_{y}\right)$ for the relationship between the two-second-order tensors, the strain tensor $\varepsilon$ and the stress tensor $\sigma$ :

$$
\boldsymbol{\sigma}=\lambda \operatorname{tr}\left(\varepsilon\left(u_{x}, u_{y}\right) \mathbb{I}+\mu \varepsilon\left(u_{x}, u_{y}\right)\right.
$$

where II denotes the 2-identity matrix,

$$
\boldsymbol{\varepsilon}=\left[\begin{array}{ll}
\varepsilon_{11}=\frac{\partial u_{x}}{\partial x} & \varepsilon_{12}=\frac{1}{2}\left(\frac{\partial u_{x}}{\partial y}+\frac{\partial u_{y}}{\partial x}\right) \\
\varepsilon_{21}=\frac{1}{2}\left(\frac{\partial u_{y}}{\partial x}+\frac{\partial u_{x}}{\partial y}\right) & \varepsilon_{22}=\frac{\partial u_{y}}{\partial y}
\end{array}\right]
$$

and $\operatorname{tr}(\cdot)$ represents the trace of a matrix. The Lamé positive constants $\lambda$ and $\mu$ are given by (cf [16])

$$
\mu=\frac{E}{(1+\nu)} \text { and } \lambda=\frac{E \nu}{(1+\nu)(1-2 \nu)},
$$

with the Young's modulus $E>0$ and the Poisson ratio $\nu \in] 0,1 / 2[$.

The time integration will be done by using a backward Euler method which is written as:

$$
\begin{aligned}
& \int_{\Omega} \frac{s^{\tau+1}}{h} w d \Omega=\int_{\Omega} \frac{s^{\tau}}{h} w d \Omega+\int_{\Omega} F\left(c^{\tau+1}, s^{\tau+1}\right) w d \Omega \\
& \int_{\Omega} \frac{c^{\tau+1}}{h} w d \Omega=\int_{\Omega} \frac{c^{\tau}}{h} w d \Omega+\int_{\Omega}\left(v_{0}-v_{1} \beta-k_{c} c^{\tau+1}-F\left(c^{\tau+1}, s^{\tau+1}\right)\right) w d \Omega+ \\
& \int_{\Omega} \mathbf{D} \nabla c^{\tau+1} \cdot \nabla w d \Omega-\int_{\Gamma_{T}} D_{1} n_{x} \frac{\partial c^{\tau+1}}{\partial x} w+D_{2} n_{y} \frac{\partial c^{\tau+1}}{\partial y} w d \Gamma \\
& \int_{\Omega} \lambda \operatorname{div}\left(u_{x}^{\tau+1}, u_{y}^{\tau+1}\right) \operatorname{div}\left(v_{x}, v_{y}\right)+\mu \varepsilon\left(u_{x}^{\tau+1}, u_{y}^{\tau+1}\right): \varepsilon\left(v_{x}, v_{y}\right) d \Omega=\int_{\Omega} \gamma\left(c^{\tau+1}\right) T_{0}\left(v_{x}, v_{y}\right) \cdot \vec{e}_{x} d \Omega
\end{aligned}
$$

for the instant $\tau>0$ and a given guess $\left(c^{0}, s^{0}\right)$,where $h$ is called the step size. We remark that at each instant we obtain a new configuration for the cell due to the displacement field $\vec{u}^{\tau+1}=\left(u_{x}^{\tau+1}, u_{y}^{\tau+1}\right)$ coming from the concentration $c^{\tau+1}$. We refer [15] for theoretical questions about existence and unicity of the numerical solutions over adequate finite element spaces.

By reasons of numerical stability the equation (13 ) must be penalized with an artificial diffusion therm using a small parameter $\xi$ like that:

$$
\int_{\Omega} \frac{s^{\tau+1}}{h} w d \Omega=\int_{\Omega} \frac{s^{\tau}}{h} w d \Omega+\int_{\Omega} F\left(c^{\tau+1}, s^{\tau+1}\right) w d \Omega+\xi \int_{\Omega} \nabla s^{\tau+1} \cdot \nabla w d \Omega
$$

The numerical scheme can be defined by:

$$
\left\{\begin{array}{l}
\text { Given a given guess }\left(c^{0}, s^{0}\right) \\
\text { determine }\left(c^{\tau+1}, s^{\tau+1}, \vec{u}^{\tau+1}\right), \tau>0 \\
\text { by solving eqs }(16),(14) \text { and }(15) \text { resp. }
\end{array}\right.
$$

We consider the cycle ends when the quantity of calcium is low in the cell: $\int_{\Omega} c^{\tau+1} d \Omega<\epsilon$, for a small $\epsilon$. 


\section{Numerical results}

At this section we will apply scheme (17) with the parameters from Table 1 using the partial differential equation solver, using finite element methods, FreeFem++ [17] .

Table 1. Cell parameters values [11]

\begin{tabular}{|c|c|c|c|c|c|}
\hline$v_{0}$ & 1 & $\mu \mathrm{M} \cdot s^{-1}$ & $K_{R}$ & 2 & $\mu \mathrm{M}$ \\
\hline$k$ & 10 & $\mathrm{~s}^{-1}$ & $K_{A}$ & 0.9 & $\mu \mathrm{M}$ \\
\hline$k_{f}$ & 1 & $\mathrm{~s}^{-1}$ & $m=n$ & $n=2$ & \\
\hline$v 1$ & 7.3 & $\mu \mathrm{M} \cdot s^{-1}$ & $p$ & 4 & \\
\hline$M_{1}$ & 65 & $\mu \mathrm{M} \cdot s^{-1}$ & $E$ & $10^{5}$ & $\mathrm{~Pa}$ \\
\hline$M_{2}$ & 500 & $\mu \mathrm{M} \cdot s^{-1}$ & $\nu$ & 0.49 & \\
\hline$K_{2}$ & 1 & $\mu \mathrm{M}$ & $T_{u}$ & $18.5 \times 10^{4}$ & $\mathrm{~Pa}$ \\
\hline
\end{tabular}

With the purpose of fulfilling a wide range of model cases, we created four different models of cardiomyocytes, varying the possible zones to place the mathematical boundary conditions for the simulation of the T-tubules, as well as the freedom of each side of the cell, in order to obtain different kinds of contraction movements. By symmetric reasons, we describe the four case as the following:

1. Case 1: T-tubule is placed on the left side of the cell, and the right side is free to contract, Figure 4 a).

2. Case 2: T-tubules is placed on the left side and both sides are free for the contraction movement, Figure $4 \mathrm{~b}$ ).

3. Case 3: T-tubule is placed in the right side and both sides are free for the contraction movement, Figure $4 \mathrm{c}$ ).

4. Case 4: Both sides are free for the contraction movement, but there are T-tubules from the upper boundary and from the bottom boundary, Figure $4 \mathrm{~d}$ ).

For all cases we simulate only one cycle, supposing a conveyable conditions of ATP and oxygen.

a)

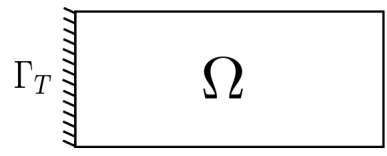

c)

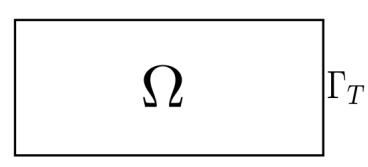

b)

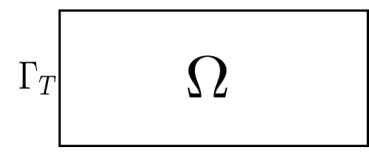

d)

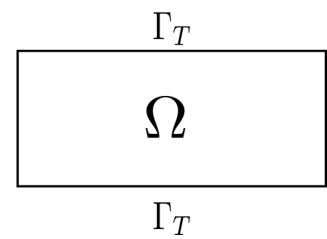

Figure 4. Outline of cardiomyocyte with different localizations for $\Gamma_{T}$

To better explain the results, we will present the graphic of calcium concentration over time, maximum cardiomyocyte contraction and the gradient of calcium diffusion for each case. 
For all exhibited graphics we have used the time $\tau$ as unity for the $\mathrm{x}$-axis and in the $\mathrm{y}$-axis we will consider calcium concentration $\mu \mathrm{M}$ for the blue graphic and percent (\%) of shortening for the green one.

The numerical scheme (17) is initiated with $\left(c^{0}, s^{0}\right)=(0.1,0.5)$ for all following cases.

\subsection{Case 1}

In this case, as described before, T-tubule is placed on the left side of the cell, and the right side is free to contract. The cardiomyocyte's contraction happens when the calcium concentration is greater than $0.45 \mu \mathrm{M}$, approximately, and we remark with Figure 5 that the contraction peak time is approximately in one-third of the cycle as it was expected.

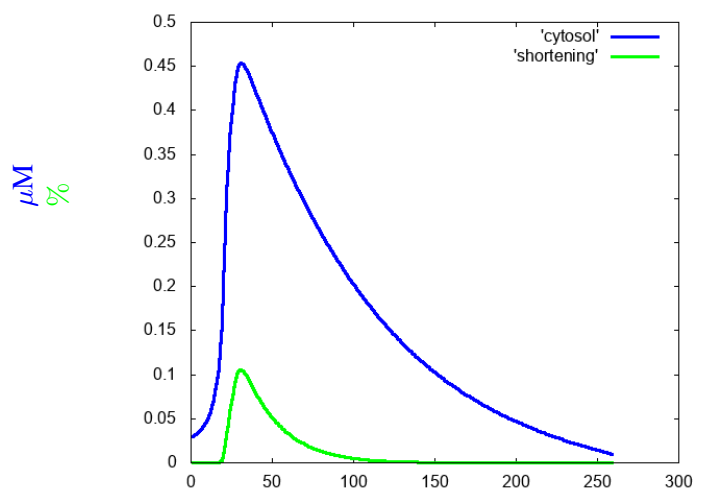

$\mathrm{ms}$

Figure 5. Cytosol concentration and shortening $\%$, during a cycle, for a $T_{u}=18.5 \times 10^{4}$ in Case 1

The maximum cardiomyocyte contraction is achieved at $\tau=41 \mathrm{~ms}$ (millisecond), as we show in Figure 6 . The initial cell length is $10 \mu \mathrm{m}$, and after contraction its length is about $8.9 \mu \mathrm{m}$, which results in a reduction of $11 \%$ (\% of shortening), s shown at Figure 6.

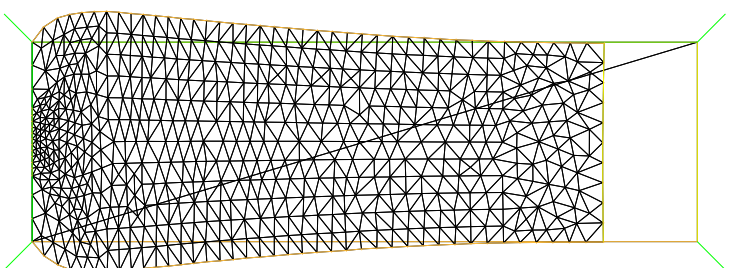

Figure 6. Maximum cardiomyocyte contraction at $\tau=41 \mathrm{~ms}$, in Case 1

The simulation of the T-tubule effect is exhibited in Figure 7 with the gradient of the calcium concentration $c^{\tau}, \tau=41 \mathrm{~ms}$.

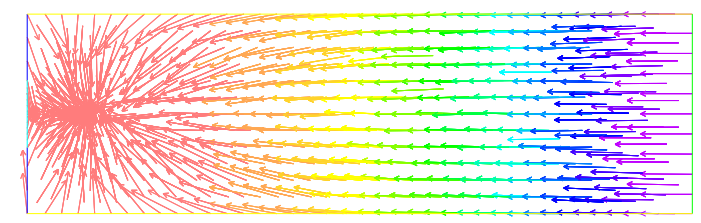

Figure 7. Gradient of the calcium concentration $\tau=41 \mathrm{~ms}$, in Case 1 


\subsection{Case 2}

In this case 2, T-tubule is also placed on the left boundary of the cell, but both left and right sides are free to contract. The cardiomyocyte's contraction happens when the calcium concentration is greater than $0.45 \mu \mathrm{M}$, approximately, and we can remark with Figure 8 that the contraction peak time is approximately one-third of the cycle as it was theoretically expected.

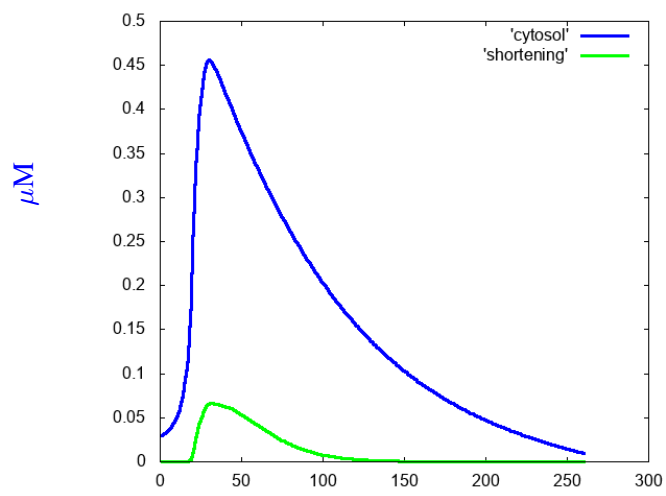

ms

Figure 8. Cytosol concentration in the cell for one cycle, in Case 2

The maximum cardiomyocyte contraction is achieved at $\tau=41 \mathrm{~ms}$, as we show in Figure9. At this case the cell length after contraction is about $9.4 \mu \mathrm{m}$, which results in a reduction of $6 \%$.

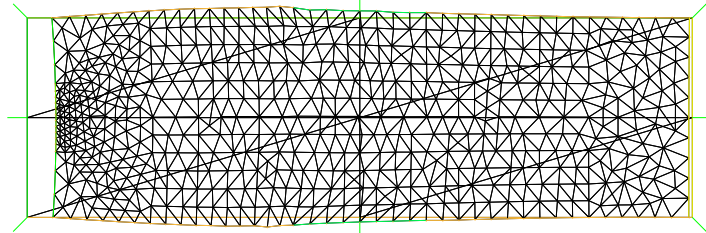

Figure 9. Maximum cardiomyocyte contraction at $\tau=41 \mathrm{~ms}$, in Case 2

The simulation of the T-tubule effect is exhibited in Figure 10 with the gradient of the calcium concentration $c^{\tau}, \tau=41 \mathrm{~ms}$.

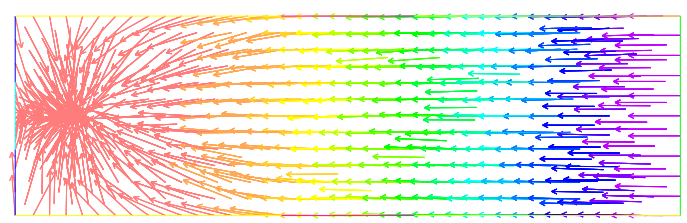

Figure 10. Gradient of the calcium concentration $\tau=41 \mathrm{~ms}$, in Case 2

\subsection{Case 3}

In this case 3, T-tubule is placed on the right side of the cell, and both left and right sides are free to contract. The cardiomyocyte's contraction happens when the calcium concentration is greater than $0.45 \mu \mathrm{M}$, approximately, and we can remark with Figure 11 that the contraction peak time is in approximately one-third of the cycle as it was expected. 


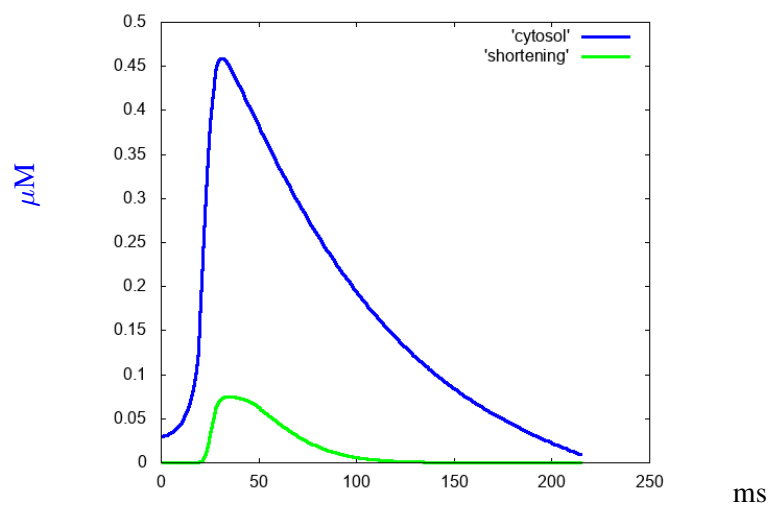

Figure 11. Cytosol concentration in the cell for one cycle, in Case 3

The maximum cardiomyocyte contraction is achieved at $\tau=41 \mathrm{~ms}$, as we show in Figure 12. As above the initial cell length is $10 \mu \mathrm{m}$, and after contraction its length is about $9.3 \mu \mathrm{m}$, which results in a reduction of $7 \%$.

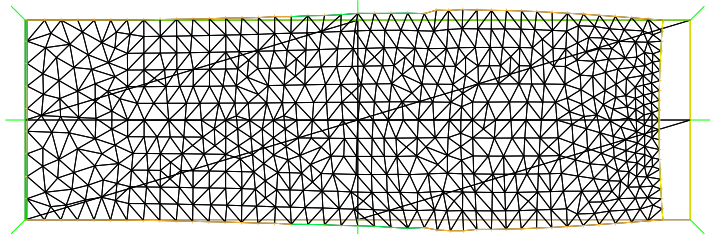

Figure 12. Maximum cardiomyocyte contraction at $\tau=41 \mathrm{~ms}$, in Case 3

The simulation of the T-tubule effect is exhibited in Figure 13 with the gradient of the calcium concentration $c^{\tau}, \tau=41 \mathrm{~ms}$.

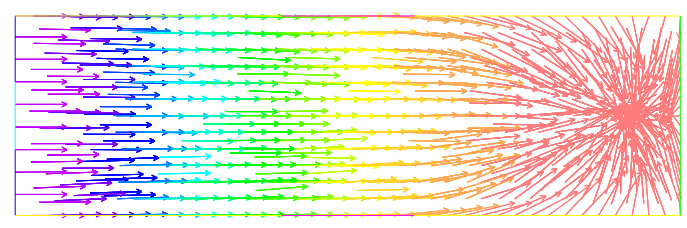

Figure 13. Gradient of the calcium concentration $\tau=41 \mathrm{~ms}$, in Case 3

\subsection{Case 4}

In this case 4, T-tubule is placed, over a reduced length zone, on the top and bottom boundaries of the model (Figure $4 \mathrm{~d})$ ) and both left and right sides are free to contract. The cardiomyocyte's contraction happens when the calcium concentration is greater than $0.36 \mu \mathrm{M}$, approximately, and we can remark with Figure 14 that the contraction peak time appears in the middle of the cycle.

The maximum cardiomyocyte contraction is achieved at $\tau=82 \mathrm{~ms}$, as we show in Figure 15. After contraction the cell length is about $9.8 \mu \mathrm{m}$, which results in a reduction of $2 \%$.

The simulation of the T-tubule effect is exhibited in Figure 16 with the gradient of the calcium concentration $c^{\tau}, \tau=82 \mathrm{~ms}$.

Finally we resume at Table 2 the main results from the study cases. 


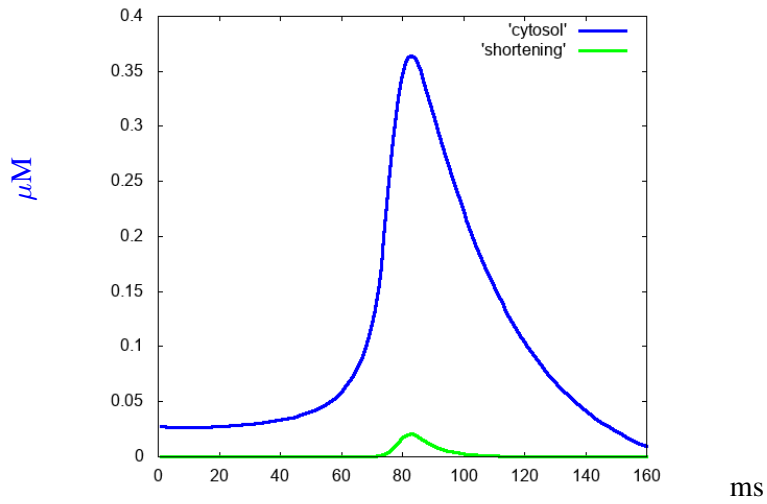

Figure 14. Cytosol concentration in the cell for one cycle, in Case 4

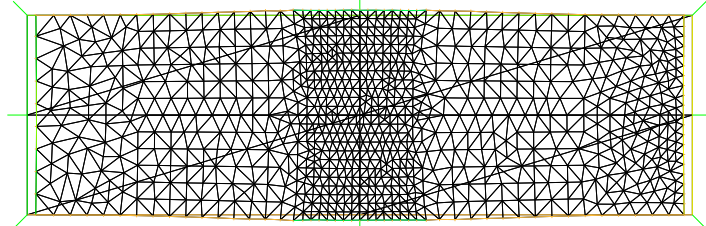

Figure 15. Maximum cardiomyocyte contraction at $\tau=82 \mathrm{~ms}$, in Case 4

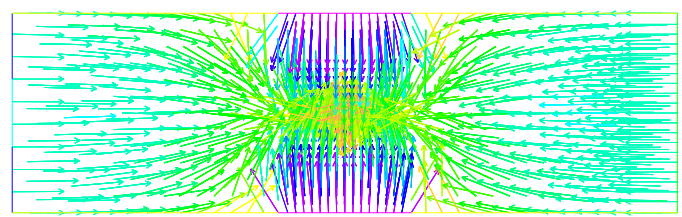

Figure 16. Gradient of the calcium concentration $\tau=82 \mathrm{~ms}$, in Case 4

Table 2. Main results comparison for study cases

Case time (ms) shortening (\%) calcium concentration $(\mu \mathrm{M})$

\begin{tabular}{llll}
1 & 41 & 11 & 0.45 \\
\hline 2 & 41 & 6 & 0.45 \\
\hline 3 & 41 & 7 & 0.45 \\
\hline 4 & 82 & 2 & 0.36 \\
\hline
\end{tabular}




\section{Conclusion}

We have investigated an Finite Element Method approach for simplified coupled mathematical model of excitationcontraction in isolated cardiomyocytes.

We started with the variational formulation model and proceed its numerical implementation based on software FreeFem++.

The results from the study cases highlight to the T-tubules distribution importance along the cardiomyocyte. We remark that for all cases the cell deformation is higher near the T-tubule boundary, as the calcium concentration. Table 2 points to the importance of a large zone for calcium influx and a existence of sides free to contract, this simulate the contact between two cells.

For case 1 , the result was a concentration of $0.45 \mu \mathrm{M}$ and a $\%$ of shortening around $10 \%$ corresponds to the results obtained by DelMonte et al [18] and Høydal et al [19]. These results are the ones expected for the physiological function of a real cardiomyocyte. This means that this simulation is reliable for these two parameters, concentration and $\%$ of shortening.

In case 2, the result was a concentration of $0.45 \mu \mathrm{M}$ and a $\%$ of shortening around $5 \%$ corresponds to the results obtained by Kamgoue et al (3\%) [20]. These results can also be considered normal or indicate a situation of hypocalcemia $(3 \%)$.

The results of case 3 were presented with a concentration of $0.45 \mu \mathrm{M}$ and a $\%$ of shortening from $4 \%$ to $10 \%$, which corresponds to the results obtained both by Kamgoue et al [20] and Garcia-Canadilla et al (9\%) [21].

At last, for case 4, with the special location of T-tubules (on the top and bottom boundarys), we reach a concentration of cytoplasmatic calcium of $0.36 \mu \mathrm{M}$ and a $\%$ of shortening of $2 \%$, which means that for this kind of model distribution (T-tubule on top and bottom), the local active cellular tension must to be higher for the same $\%$ of shortening.

The results obtained with our model are faithful to what is known to be true with cell's physiology and pathological conditions (hypocalcemia).

This study proposes a simplification of the mathematical context of the excitation-contraction in isolated cardiomyocytes problem and brings a contribution to a very complex subjet.

A relevant study is to introduce in this mathematical model multiple parametric, to enable understanding its influence, namely the ones that are considered to be important to studied cardiac diseases.

\section{Acknowledgement}

This research was supported by project IGACFC, IDI\& CA-IPL 2018.

\section{REFERENCES}

1. U. Primessnig, P. Schönleitner, and A. Höll, Novel pathomechanisms of cardiomyocyte dysfunction in a model of heart failure with preserved ejection fraction, European Journal of Heart Failure (2016) 18, 987-997.

2. P. Gorski2015, D. Ceholski, and R. Hajjar Altered Myocardial Calcium Cycling and Energetics in Heart Failure - A Rational Approach for Disease Treatment, Cell Metabolism 21,pp. 183-194, 2015.

3. N. Sperelakis, Y. Kurachi, A. Terzic, and M. Cohen, Heart Physiology and Pathophysiology - 4th Edition, Academic Press, 2001.

4. K. Barrett, S. Barman, S. Boitano, and H. Brooks, Ganong's Review of Medical Physiology, 25th Ed. McGraw-Hill Education

5. D. Eisner, J. Cadwell, K. Kistamás and W. Trafford, Calcium and Excitation-Contraction Coupling in the Heart, Circulation Research, 121(2):pp. 181-195, 2017.

6. M. Jafri, Models of Excitation-Contraction Coupling in Cardiac Ventricular Myocytes, Methods Mol Biol. 2012 ; 910: $309-335$. doi:10.1007/978 - $1-61779-965-5{ }_{1} 4$.

7. R. Klaubunde, Cardiac Excitation-Contraction Coupling, Cardiovascular Physiology Concepts - www.cvphysiology.com.

8. L. Vilaça, Mathematical Modeling and Numerical Simulation of Excitation-Contraction Phenomena in the Heart, Departement of Chemistry in EPFL, 2013

9. G. Ikonnikov, and E. Wong, Action potentials and impulse conduction, www.pathophys.org

10. N. El-Sherif, and G. Turitto, Electrolyte disorders and arrhythmogenesis, Cardiology Journal, Vol. 18, No. 3, pp. 233-245, 2011.

11. A. Goldbeter, G. Dupont, and M. Berridge, Minimal model for signal-induced Ca2+oscillations and for their frequency encoding through protein phosphorylation., Proceedings of the National Academy of Sciences of the United States of America, 1990. 
12. A. Beck, and M. Teboulle, A fast iterative shrinkage-thresholding algorithm for linear inverse problems, SIAM Journal on Imaging Sciences, vol. 2, no. 1, pp. 183-202, 2009

13. S. Subramanian, S. Viatchenko-Karpinski, V. Lukyanenko,S Györk and T.F. Wiesner, Underlying mechanisms of symmetric calcium wave propagation in rat ventricular myocytes, Biophysical Journal,80(1), pp. 1-11, 2001.

14. O. Cazorla, A. Lacampagne, J. Fauconnier, and G. Vassort, , SR 33805 a Ca $^{2+}$ antagonist with lengtdependent Ca ${ }^{2+}{ }_{-s e n s i t i z i n g}$ properties in cardiacmyocytes, Brit. J. Pharmacol., 139, pp 99-108, 2003.

15. Joseph D. Bronzino, eds., Biomedical Engineering Handbook Vol 1, Springer-Verlag Berlin and Heidelberg GmbH \& Co. K ,2000.

16. C. Johnson, Numerical Solution of Partial Differential Equations by the Finite Element Method, Springer-Verlag Berlin and Heidelberg GmbH \& Co. K ,2000.

17. F. Hecht, New development in FreeFem++, Journal of Numerical Mathematics, 20 (3-4), pp. = 251-265, 2012.

18. F. del Monte, and S. Harding, and U. Schmidt, and T. Matsui, and et al, Restoration of Contractile Function in Isolated Cardiomyocytes From Failing Human Hearts by Gene Transfer of SERCA2a, Circulation.,23 (100), pp 2308-2311, 1999.

19. M. Høydal, and I. Kirkeby-Garstad, and A. Karevold, and R. Wiseth, and et al., Human cardiomyocyte calcium handling and transverse tubules in mid-stage of post-myocardial-infarction heart failure, ESC Hear. Fail., February, pp 332-342, 2018, doi = $10.1002 /$ ehf 2.12271 .

20. A. Kamgoué, and J. Ohayon, and Y. Usson, and L. Riou, and P. Tracqui, Quantification of cardiomyocyte contraction based on image correlation analysis, Cytom. Part A, 4(75), pp 298-308,2009, doi = 10.1002/cyto.a.20700.

21. P. Garcia-Canadilla, and J. Rodriguez, and MJ. Palazzi, and A. Gonzalez-Tendero, and et al., A two dimensional electromechanical model of a cardiomyocyte to assess Intra-cellular regional mechanical heterogeneities PLoS One, 8(12), pp 1-20, 2017, doi = 10.1371/journal.pone.0182915. 